\title{
Fastening of shape memory hook arrays
}

\author{
D. Vokoun ${ }^{1, \mathrm{a}}$, D. Majtás ${ }^{1}$, M. Frost ${ }^{1,2}$, P. Sedlák ${ }^{2}$ and P. Šittner ${ }^{1}$ \\ ${ }^{1}$ Institute of Physics ASCR, v.v.i., Na Slovance 2, 18200 Prague 8, Czech Republic \\ ${ }^{2}$ Institute of Thermomechanics ASCR, v.v.i., Dolejškova 1402/5, 18200 Prague 8, Czech Republic
}

\begin{abstract}
Shape memory (SM) fasteners based on the SM effect are already well-known applications. However, it has just been recently that NiTi hook arrays working as probabilistic fasteners were fabricated. Similarly to Velcrotype fasteners our fasteners are releasable with hooks returning back to their original shape after releasing. NiTi hooks may be incorporated into textiles and function in the same manner as Velcro fasteners do or they may be incorporated into a rigid substrate. The main objective of our research is twofold: First, we would like to show the influence of the material properties and geometry parameters of pseudoelastic NiTi micro-hooks on the interlocking force necessary for releasing the NiTi hook connection. Secondly, we show a method for aligning of the micro-hooks into an array and attaching them to a substrate.
\end{abstract}

\section{Introduction}

Velcro-type fasteners are still receiving much attention as a bio-inspired application [1]-[3]. In the present study we combine material and geometrical properties of metal hooks on the basis of study of two distinct subjects: Probabilistic fastening and shape memory. It has been suggested earlier that shape memory (SM) hooks may be used as Velcro-type fasteners [4]. However, the authors of [4] did not bring the idea to a real application and to our knowledge their research was not continued further. It has been just recently we succeeded to produce a pseudoelastic Velcro-type fastener [5] made of NiTi wire hooks and a rigid substrate. In [5] we introduced the NiTi hook fastener manufacture including a simple mathematical model predicting the minimum force necessary for unlocking of two pseudoelastic NiTi hooks depending on geometric and material parameters of the two interlaced hooks.

In our research of Velcro-type fasteners we utilize the pseudoelastic effect of NiTi wires. During the fastener releasing by pulling apart of the two hook arrays each individual hook is bent, having large deformations at its surface. Due to the pseudoelastic behavior of NiTi hooks the large deformations are recovered upon hook releasing. Another advantage of NiTi hooks in Velcro-type fasteners is less noise (due to damping capacities of SMAs) during the hook release when compared to the release action of the typical Velcro fasteners. Furthermore, other advantages such as better durability, heat resistance, or functioning in chemically aggressive environments can be appreciated.

In the present study, we first describe the Velcro-type fastener manufacture that is different from that described in [5]. In the second part, we summarize the previously presented 1D model first introduced in [5] and apply the model for arrays of NiTi hooks to obtain minimum pulling force necessary for separating two interlocked hook arrays. The mechanical experiments done with Velcro-type fasteners are described including the measurement of the normal pulling force.

\section{Experimental}

\subsection{NiTi micro-hook shape-setting}

A cold drawn NiTi wire with a diameter of $0.1 \mathrm{~mm}$ purchased from a commercial company was shape-set using a thin steel plate (Fig. 1). The as-drawn wire was tightly wound around the steel plate of a ribbon-like shape. More than 1000 wire loops were formed. Loosening of the wire was prevented by fixing both the wire ends with screws. The steel plate is about $0.7 \mathrm{~mm}$ thick, $6 \mathrm{~mm}$ wide and more than $100 \mathrm{~mm}$ long. After winding, the plate together with the wire was put in a furnace for annealing at $450^{\circ} \mathrm{C}$ for 30 minutes. The individual hooks were then cut from the shape-set superelastic wire structure. The hook curvature given by the plate thickness turned

\footnotetext{
a e-mail : vokoun $a$ ffiı.c7
} 
out to be crucial for thermomechanical behavior of the hooks. The hooks were further manufactured into SMA hook arrays.

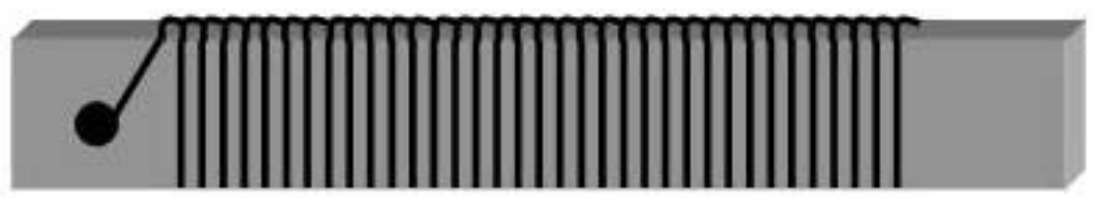

Fig. 1 A scheme of a fixture for shape-setting of the SMA hooks.

\subsection{Hook arrays, manufacture and the measurement}

Our aim is to manufacture NiTi hook arrays, with the following requirements:

(i) All the hook's straight ends are mutually parallel and perpendicular to the rigid matrix.

(ii) All the hook's tops are at the same height above the rigid matrix surface.

(iii) All the hooks are spread evenly at the nods of a regular grid.

(iv) The hook's axial rotation in respect to the grid is random.

As for achieving (i)-(iv), two steel plates with the dimensions $22 \mathrm{~mm}$ × $22 \mathrm{~mm}$ × $2 \mathrm{~mm}$ and an array of microchannels of a diameter $0.2 \mathrm{~mm}$ laser-drilled in the plates were prepared. Three microchannel densities realized were 121, 441, and 961 holes per one plate. The holes were evenly arranged in a regular grid in the square area of $4 \mathrm{~cm}^{2}$. Before hook insertion, the holes were filled with clay to prevent liquid leaking. The array of inserted hooks was aligned so that each hook top was $2.5 \mathrm{~mm}$ above the plate's surface. Then, the aligned side of the hooks (hook heads) was dipped in a liquid layer of 2-component Si based polymer. After polymerization (12 hours at room temperature) all the hook heads were embedded in the rubber-like polymer layer and the metal matrix was removed. The hook's ends were cleaned in acetone and later cast in epoxy resin. After resin curing at temperature $80^{\circ} \mathrm{C}$ for 2 hours the resin became rigid. Since the resin layer does not adhere to the rubber layer, the rubber layer can be peeled off. During peeling off, the hooks are temporarily deformed but due to the pseudoelastic effect they recover their original shape. In this way the SMA hook array samples were ready for the mechanical experiments. The experimental setup is shown in Fig. 2.

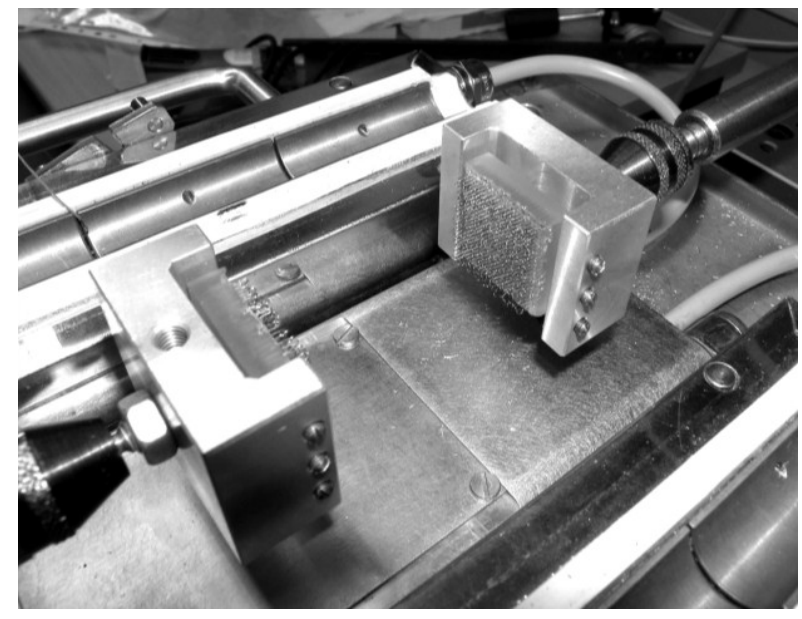

Fig. 2 A photograph of loading of the NiTi hook arrays

Since we produced hook array fasteners of three various densities of NiTi hooks, three series of mechanical tests were carried out, each with different array density. In each series the interlocked SMA hook arrays were pulled apart along the axis that is perpendicular to both the array planes while force was measured until complete SMA hook release. In the first (Fig. 3a) [second (Fig. 3b) and third (Fig. 3c)] series of experiments we used the hook arrays with the density 30 hooks $/ \mathrm{cm}^{2}\left[110\right.$ hooks $/ \mathrm{cm}^{2}$ and 240 hooks $\left./ \mathrm{cm}^{2}\right]$, respectively. Each diagram of Fig 3 shows just two experimental curves of 12 carried out ones - the lowest and the highest curves. 
Since the number of the interlocked hooks is variable in each experiment, the loading curves in each diagram of Fig. 3 are not overlapping. The maximum force lies inside a certain force interval in each series and the largest maximum force values are attained in the third series using SMA hook arrays with the density of 240 hooks $/ \mathrm{cm}^{2}$. The maximum force intervals in series 1,2 , and 3 are $0.62 \mathrm{~N}-1.50 \mathrm{~N}, 2.86 \mathrm{~N}-7.77 \mathrm{~N}$ and $38.55 \mathrm{~N}$ $-50.59 \mathrm{~N}$.
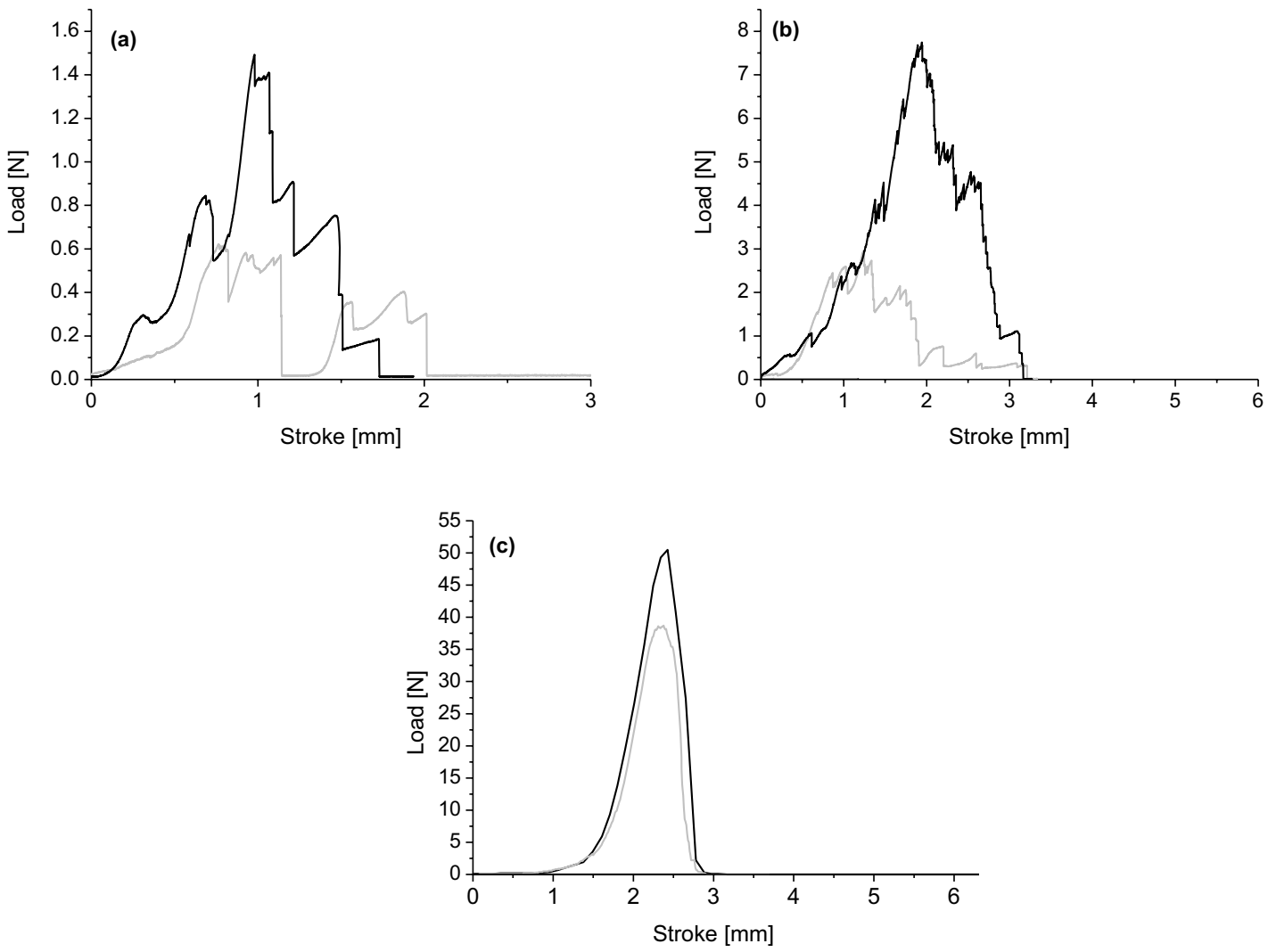

Fig. 3 A diagram of hook load versus stroke for SMA hook arrays with various densities of hooks: (a) 30 hooks $/ \mathrm{cm}^{2}$, (b) 110 hooks $/ \mathrm{cm}^{2}$, (c) 240 hooks $/ \mathrm{cm}^{2}$.

\section{Model}

Our model was created as an answer to our need for determining the minimum force necessary for SMA hook releasing. However, the created model is additionally capable to simulate the whole load - stroke curve for SMA hooks of any geometrical and material parameters. The model is already described in [5], therefore we mention only the most important features of the model.

For the description of pseudoelastic behavior we used a part of the phenomenological 1D model iRLOOP [6]. This part referred to as module of the iRLOOP model has the following features:

- $\quad$ supports the concept of thermodynamic force coupling stress and temperature, which drives the transformation processes,

- reliably describes both the entire and partial martensite to austenite and reverse transformation processes with partial cycles,

- captures asymmetric behavior in tension and compression in pseudoelasticity,

- $\quad$ is able to be driven by prescribed evolution of strain and temperature of a wire,

- $\quad$ is able to adapt to particular material by changing several experimentally well-measurable input parameters,

The model introduces two internal variables - tensile martensite volume fraction, $\xi^{+}$, and compressive martensite volume fraction, $\xi$. Each value of tensile (compressive) martensite volume fraction depends on the driving 
force for martensitic transformation, $\phi^{+}\left(\phi^{-}\right)$. The envelope functions of the hysteretic loop (volume fraction driving force) are approximated by piece-wise linear functions which best fit the experimental data (Fig. 4). Since tensile and compressive martensite never appear simultaneously for temperature $T>A_{f}$ (austenite finish), which is the case of our study, we can work with the evolutions of $\xi^{+}$and $\xi$ as two completely independent functions.

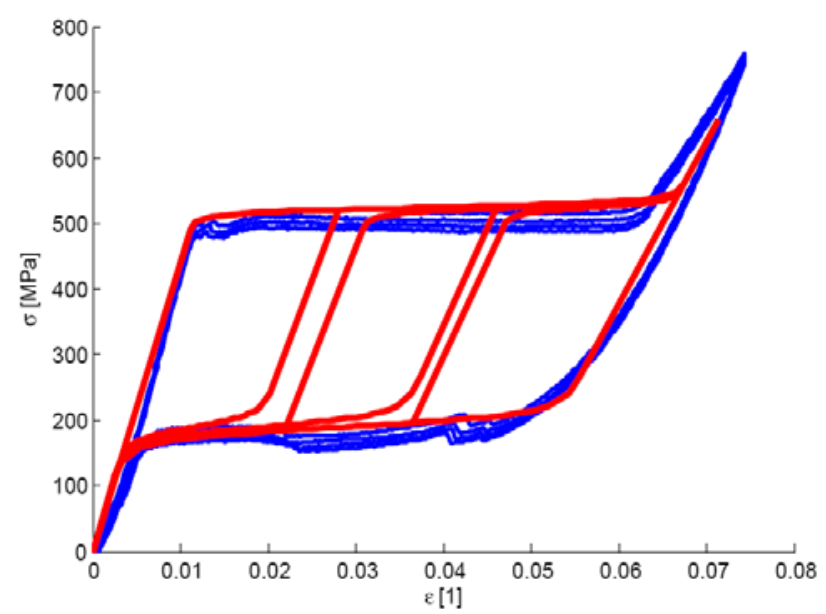

Fig. 4 A diagram of tensile stress-strain curve (a) measured at room temperature for the annealed wire (blue) and (b) modeled tensile stress-strain curve used as an input of the NiTi hook model (red).

In the simulation of the SMA hook loading, the hook was divided to a system of beam elements. We assume that during bending the normal lines to the cross section areas of the beam remain parallel to the beam axis. Furthermore, the superelasticity module of iRLOOP was used for each of the beam elements.

The boundary conditions are given by the fixation of the straight end of the hook (fixation of displacement and rotation) and by placing the loading force to the point, where the force direction is perpendicular to the beam axis tangent line. Any friction is neglected. Figure 5 and Fig. 6 show the geometrical parameters and the modeled pulling force - stroke curve for a single hook, respectively. The maximum pulling force of a single NiTi hook is $0.24 \mathrm{~N}$.

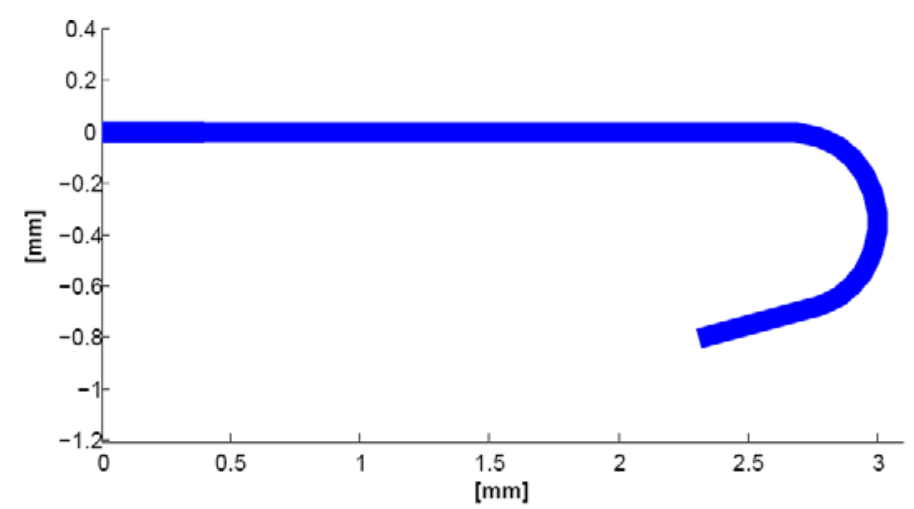

Fig. 5 Geometrical parameters of a single NiTi hook.

If we know the mean number of interlocked hooks in the fasteners, we are able to estimate the maximum pulling force in the diagrams of pulling force versus stroke in Fig. 3. For the determination of the number of interlocked hooks for given hook density and hook geometry we used a kind of Monte-Carlo simulations. We assume the hooks are randomly rotated around their long axis and both the hook arrays are mutually shifted by a small random value. Without going into details, we obtained the mean numbers of interlocked hooks summarized in Table 1. 


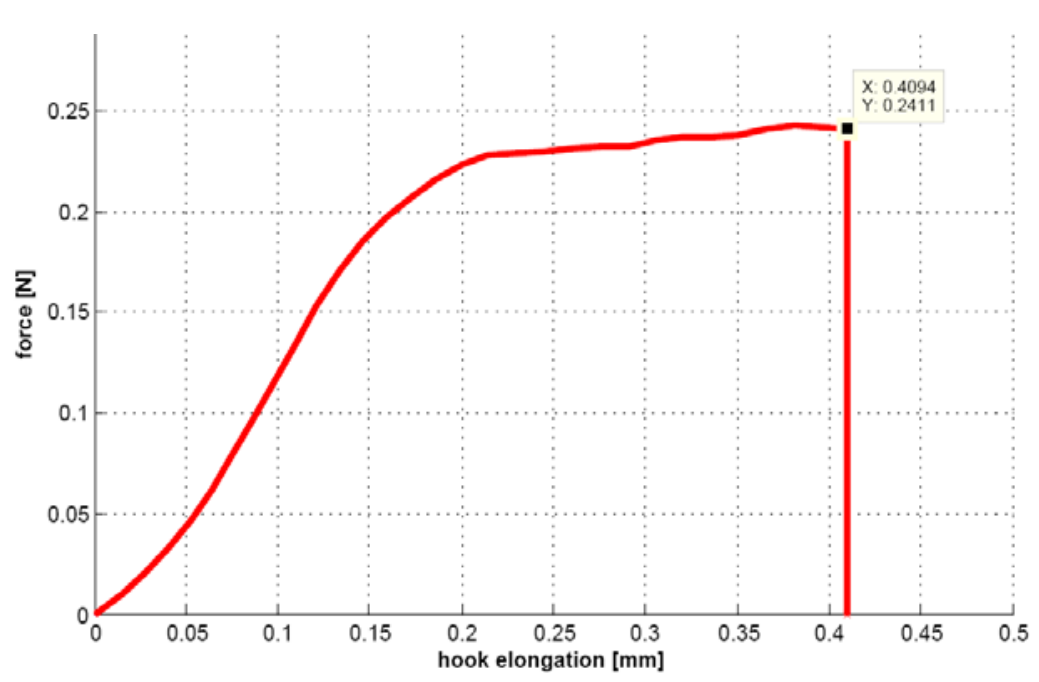

Fig. 6 The diagram of the modeled normal pulling force versus stroke.

Table 1. Mean numbers of interlocked hooks for various hook densities and the corresponding estimated maximum pulling force.

\begin{tabular}{|c|c|c|c|}
\hline $\begin{array}{c}\text { Number of all hooks in a } \\
\text { sample }\end{array}$ & $\begin{array}{c}\text { Hook density } \\
\left.\text { [hook number } / \mathrm{cm}^{2}\right]\end{array}$ & $\begin{array}{c}\text { Mean number of } \\
\text { interlocked hooks }\end{array}$ & $\begin{array}{c}\text { Corresponding estimated } \\
\text { maximum pulling force } \\
{[\mathrm{N}]}\end{array}$ \\
\hline 121 & 30 & 7 & 1.68 \\
\hline 441 & 110 & 116 & 27.84 \\
\hline 961 & 240 & 525 & 126.0 \\
\hline
\end{tabular}

\section{Discussion}

It is obvious from Table 1 that the estimate of the maximum pulling force is larger than the measured maximum force for all three densities. There are several reasons for the overestimation of the maximum pulling force: (i) The hooks may not be well aligned which results in gradual releasing of the interlocked hooks. This phenomenon may be observed in Fig. 3a where the curves have several peaks. (ii) During the experiment, the sample substrates may not be exactly parallel. (iii) There may exist hooks interlocked with two and more hooks (i.e. a different boundary condition than we assumed).

In our future work, we plan to measure the pulling force of a single hook to verify that the maximum force of a single hook is close to the modeled value of $0.24 \mathrm{~N}$. We have not done it so far because our load cell is not suitable for small force measurements. Furthermore, we plan to assume a certain distribution of the hook's length and a certain mutual tilt of the sample substrates. Such a model will help us to find optimum material and geometry parameters without the need of manufacturing many samples of various parameters.

\section{Conclusions}

(1) A procedure for shape-setting NiTi hooks and making SMA hook arrays is shown.

(2) The model enables us the prediction of the mechanical response of a single NiTi hook. The maximum pulling force of the whole hook array is estimated based on the mean number of interlocked hooks. However, our model overestimates the maximum pulling force. The reasons are mentioned above.

(3) We can see clearly from the measurements and modeling that the maximum pulling force is increasing with increasing hook density. 


\section{Acknowledgment}

D.V.and D.M. acknowledge support by a Marie Curie International Reintegration Grant within the 6th European Community Framework Programme.

\section{References}

[1] S. Berber, Y.K. Kwon and D. Tomanek. Phys. Rev. Letters 91 (2003) 165503-1.

[2] J.A. Williams, S.G. Davies, S. Frazer, Tribology letters 26 (2007): 213-222

[3] S.N. Gorb, V.L. Popov, Philosophical Trans. of the Royal Soc of London A-Mathematical and Phys. Engineering Sciences 360 (2002) Pages: 211-225.

[4] A. Schuster. Dissertation zur Erlangung des Grades Doktor-Ingenier. Der Fakultat fur Maschinenbau der Ruhr-Universitat Bochum. Bochum 2001.

[5] D. Vokoun, D. Majtás, M. Frost, P. Sedlák and P. Šittner. J of Mater Eng and Performance. In Press.

[6] M. Frost, M., Sedlák, P., Sippola, M., Šitner, P.: Smart Mater. Struct., in review 